\title{
LIM homeodomain transcription factor Is/1 affects urethral epithelium differentiation and apoptosis via Shh
}

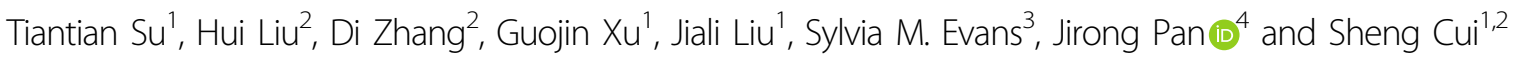

\begin{abstract}
Urethral hypoplasia, including failure of urethral tube closure, is one of the common phenotypes observed in hereditary human disorders, the mechanism of which remains unclear. The present study was thus designed to study the expression, functions, and related mechanisms of the LIM homeobox transcription factor Is/1 throughout mouse urethral development. Results showed that $/ s / 7$ was highly expressed in urethral epithelial cells and mesenchymal cells of the genital tubercle (GT). Functional studies were carried out by utilizing the tamoxifen-inducible $/ s / 7$-knockout mouse model. Histological and morphological results indicated that $/ s / 1$ deletion caused urethral hypoplasia and inhibited maturation of the complex urethral epithelium. In addition, we show that Is/1-deleted mice failed to maintain the progenitor cell population required for renewal of urethral epithelium during tubular morphogenesis and exhibited significantly increased cell death within the urethra. Dual-Luciferase reporter assays and yeast one-hybrid assays showed that ISL1 was essential for normal urethral development by directly targeting the Shh gene. Collectively, results presented here demonstrated that Is 11 plays a crucial role in mouse urethral development, thus increasing our potential for understanding the mechanistic basis of hereditary urethral hypoplasia.
\end{abstract}

\section{Introduction}

External genital development of mammals is regulated by a sophisticated progression of budding and fusion events. In mice, paired genital swellings arise lateral to the cloacal membrane at around embryonic day (E) 10.5, then these swellings merge to form a single genital tubercle (GT) at E11.5, and finally give rise to the prepuce by E13.5. Up to E15.5, male and female GTs are morphologically indistinguishable, and their development is presumably controlled by the same genetic program called androgen-independent GT patterning ${ }^{2-4}$. After E16.5,

\footnotetext{
Correspondence: Jirong Pan (panjirong@cnilas.org) or

Sheng Cui (cuisheng@cau.edu.cn)

${ }^{1}$ State Key Laboratory of Agrobiotechnology, College of Biological Sciences, China Agricultural University, 100193 Beijing, People's Republic of China

${ }^{2}$ College of Veterinary Medicine, Yangzhou University, 225009 Yangzhou, Jiangsu, People's Republic of China

Full list of author information is available at the end of the article

These authors contributed equally: Tiantian Su, Hui Liu

Edited by I. Amelio
}

urethra in males canalize in the presence of androgen signaling, whereas they remain as epithelial cords in females $^{5,6}$. Three germ layers participate in external genital development: endodermally derived urethral plate epithelium forms the entire urethral tube, mesoderm forms the stromal tissue of the genital, and a jacket of ectodermal epithelium forms the $\operatorname{skin}^{7-9}$. Epithelial differentiation is an important process for reproductive organ development. Urethral defects lead to severe developmental disease; a prime example being the most common urethra defect in human, hypospadias, a failure of urethral tube closure often accompanied by agenesis of the ventral aspect of the genitals, which affects $\sim 1$ in 250 live births ${ }^{10,11}$.

Genetic mutation is one of the crucial causes of urethral defects. Up to now, several factors have been identified that participate in urethral development. Sonic hedgehog $(S h h)$ mutants result in hypospadias associated with increased cell death and failure to maintain Wnt-Fgf8

\section{(c) The Author(s) 2019}

(c) (i) Open Access This article is licensed under a Creative Commons Attribution 4.0 International License, which permits use, sharing, adaptation, distribution and reproduction in any medium or format, as long as you give appropriate credit to the original author(s) and the source, provide a link to the Creative Commons license, and indicate if changes were made. The images or other third party material in this article are included in the article's Creative Commons license, unless indicated otherwise in a credit line to the material. If material is not included in the article's Creative Commons license and your intended use is not permitted by statutory regulation or exceeds the permitted use, you will need to obtain permission directly from the copyright holder. To view a copy of this license, visit http://creativecommons.org/licenses/by/4.0/. 
signaling in endoderm ${ }^{2,7,12-15}$. The Fgf8-expressing distal urethral epithelium has been shown to have a growthpromoting function ${ }^{16,17}$. Fgfr2 is required for outgrowth and patterning of the GT, and regulates cell number by controlling the length of specific cell cycle phases ${ }^{10,18,19}$. Hoxa13 mutant hypospadias occur as a result of combined loss of $F g f 8$ and $B m p 7$ expression in the urethral epithelium $^{16}$. Genes including $\operatorname{Lef1}^{20,21}, M s x 1^{1}, \quad b$ catenin $^{22}$, and Noggin ${ }^{6}$ also have been shown to be involved in urethral development. However, a comprehensive network of gene regulation during urethral development remains unclear.

The LIM homeobox gene Isl1 plays critical roles in multiple tissues in mouse embryonic development, including the nerve $\mathrm{e}^{23,24}$, stomach $^{25,26}, \operatorname{limb}^{27,28}$, and heart ${ }^{29-32}$, by functioning in cell proliferation, apoptosis ${ }^{33}$, and differentiation ${ }^{34,35}$. ISL1 is also involved in hormone biosynthesis and secretion in endocrine tissues such as pancreatic islets ${ }^{36,37}$, pituitary $^{38}$, and pineal glands ${ }^{39,40}$. Isl1 expression has been detected in genital mesenchyme, and Isl1 knockout in mesenchyme results in urogenital malformations, although urethral development in these Isl1 mutants was unaffected ${ }^{41}$. Additionally, genome-wide association studies have revealed that Isl1 is a major susceptibility gene for human congenital anomalies of the kidney and urinary tract (CAKUT) and bladder exstrophy-epispadias complex (BEEC) $)^{42-44}$, implying potential roles for Isl1 in urethra development. The present study was thus designed to examine a potential role for the transcription factor Isl1 in urethral development.

\section{Materials and methods}

\section{Mice maintenance and treatment}

Adult (6- to 8-week-old) male and female C57BL/6 mice were used for this study. The age of embryos was determined by the appearance of the vaginal plug, which was taken to be E0.5.

Generations of $I s l 1^{M C M / D e l}$ and $I s l 1^{F /+}$ mice were described previously ${ }^{45}$. Briefly, we used a "floxed" Isl1 allele $(I s l 1 F)$ in which LoxP sites were inserted into the introns flanking exon 4 of the Isl 1 locus, and a tamoxifeninducible knock in Isl1-MERCreMER allele. Isl $1^{\mathrm{F} / \mathrm{F}}$ mice were mated with $I s l 1^{\mathrm{MCM} /+}$ mice to generate litters with equal numbers of $I s l 1^{\mathrm{MCM} / \mathrm{F}}$-inducible knockouts and $I s l 1^{F /+}$ controls. To induce excision in $I s l 1^{\mathrm{MCM} / \mathrm{F}}$ embryos, pregnant females were administered an oral gavage of $75 \mathrm{mg} / \mathrm{kg}$ body weight of tamoxifen (T5648; Sigma, St. Louis, MO, USA) in corn oil $(10 \mathrm{mg} / \mathrm{ml})$ at $\mathrm{E} 9.5$ for three consecutive days just before Isl1 expression sharply increased. Embryos were harvested from pregnant mice obtained by timed matings at the desired stages of development and genotyped by common PCR. Sex of embryos were identified by common PCR before E15.5 ${ }^{46}$ and later morphological analysis of gonads. For studies involving embryos, only males were presented (except for specially marked parts). All animal studies were approved by Ethics Committee of China Agricultural University and performed in accordance with the guidelines and regulatory standards of the Institutional Animal Care and Use of Animals for Scientific Purposes.

\section{Common PCR and qPCR}

GTs were dissected from stage-matched embryos and were pooled according to genotype for each litter collected. Total RNA was extracted using Trizol reagent (9109; TaKaRa, Dalian, China). RNA quantity and purity were determined using a NanoDrop (ND-2000, USA). One microgram of high-quality RNA (260/280 ratios slightly higher than 2.0 and 260/230 ratios higher than 1.7 for each pooled sample was reverse transcribed into complementary DNA (cDNA) using M-MLV (M170A; Promega, USA). Quantitative PCR (qPCR) amplication was performed four times (DRR420A; Takara, Dalian, China) in the ABI 7500 system (Applied Biosystems, Foster City, USA). For normalization purposes, an identical set of reactions were prepared for glyceraldehyde 3phosphate dehydrogenase (GAPDH).

Genomic DNA was isolated from tail or GT following the HotSHOT method ${ }^{47}$ and genotyping was performed using standard PCR methods with specific primers ${ }^{45}$. The relative location of primers used to identify the wild-type (WT), floxed (Flox), and rearranged alleles are shown as solid arrows in Fig. 2b; primer pair F1/R1 amplifies a 406 bp WT and 502 bp Flox bands. In $I s l 1^{\text {MCM/Del }}$ mice, the expected PCR products using the primer pairs $M C M$ $F$ and $M C M-R$ were $289 \mathrm{bp}$. Primer pair F1/R2 amplifies a 730-bp rearranged floxed allele in $I s l 1^{M C M / D e l}$ genital after Cre activation; there was no product under the same PCR condition before Cre activation. The common PCR (BioRad Laboratories) was performed using the following protocol: $95^{\circ} \mathrm{C}$ for $5 \mathrm{~min} ; 95^{\circ} \mathrm{C}$ for $30 \mathrm{~s}, 60^{\circ} \mathrm{C}$ for $30 \mathrm{~s}$, $72{ }^{\circ} \mathrm{C}$ for $45 \mathrm{~s}$ ( 35 cycles); $72{ }^{\circ} \mathrm{C}$ for $5 \mathrm{~min} ; 4^{\circ} \mathrm{C}$ holding. PCR products were observed on a $1.5 \%$ agarose gel. PCR primers designed for this study were listed in Supplementary Tables S1 and S2.

\section{Western blot}

Briefly, total proteins were extracted in RIPA (radio immunoprecipitation assay) buffer (9806; Cell Signaling, Danvers, MA, USA) containing $1 \mathrm{mM}$ phenylmethylsulfonyl fluoride (8553S; Cell Signaling, Danvers, MA, USA) according to the manufacturer's protocol. The BCA Protein Assay Kit (HX18651; Hoaxing, China) was used to measure protein concentration. Electrophoresis was performed with $30 \mu \mathrm{g}$ total proteins separated by $12 \%$ sodium dodecyl sulfate-polyacrylamide gel electrophoresis and transferred to polyvinylidene difluoride membranes (IPVH00010, Millipore, USA). The 
membrane was blocked with $5 \%(\mathrm{w} / \mathrm{v})$ nonfat dry milk in $0.05 \mathrm{M}$ Tris-buffered saline and 0.1\% Tween-20 (TBST, $\mathrm{pH}$ 7.4) for $1 \mathrm{~h}$ and incubated with anti-ISL1(1:300; AF1837; R\&D, Minneapolis, MN, USA) antibody and internal control GAPDH antibody (1:10,000; Ambion, USA) overnight at $4{ }^{\circ} \mathrm{C}$. The secondary antibody, horseradish peroxidase-conjugated donkey anti-goat IgG (1:10,000; ab205723; Abcam, Cambridge, MA, USA), was diluted 1:5000 in TBST. The membranes were visualized using the SuperSignal West Pico Kit (Thermo Scientific, Waltham, MA, USA) substrate at room temperature. We used the ImageJ Software to assay the relative intensity of each blot. The intensity values of each group were normalized to GAPDH (internal control) in the same group.

\section{Hematoxylin and eosin staining}

The embryonic specimens were fixed overnight in $4 \%$ paraformaldehyde/phosphate-buffered saline (PBS), dehydrated in ethanol, and embedded in paraffin. Sections of $5 \mu \mathrm{m}$ were cut from wax-embedded embryos and floated onto slides coated with 3-triethoxysilylpropylamine (440140, Sigma). The slides were dried at $37^{\circ} \mathrm{C}$ overnight, dewaxed through xylene, and then rehydrated through decreasing concentrations of ethanol. Next, they were stained with hematoxylin, counterstained with eosin, dehydrated, and equilibrated with xylene. Sections were photographed under bright-field microscope photograph system (Leica Microsystems, Buffalo Grove, IL, USA).

\section{Immunofluorescence and immunohistochemistry}

The sections were deparaffinized, rehydrated, and subjected to microwave antigen retrieval with $0.01 \mathrm{M}$ sodium citrate buffer $(\mathrm{pH}$ 6.0). The sections were then blocked with $10 \%$ normal donkey or goat serum in PBS at room temperature for $1 \mathrm{~h}$, followed by the incubation with antiISL1 antibody (1:50; 40.2D6; Developmental Studies Hybridoma Bank, Iowa City, IA, USA), antibromodeoxyuridine (BrdU) (1:300; G3G4; Developmental Studies Hybridoma Bank) overnight at $4{ }^{\circ} \mathrm{C}$. After rinsing thoroughly with PBS, the sections were incubated with the secondary antibodies, which were cy3conjuncted goat anti-mouse IgG (1:50; 115-165-003; Jackson ImmunoResearch, West Grove, PA, USA) for $2 \mathrm{~h}$ at room temperature. The sections were then rinsed with PBS and stained with 4',6-diamidino-2-phenylindole (10236276001; Roche Applied Science, Basel, Switzerland) for $10 \mathrm{~min}$. Finally, $20 \mu \mathrm{l}$ Vectashield mounting medium (H-1000; Vector Laboratories, Burlingame, CA, USA) was applied to each slide, and a coverslip was sealed in place. Leica Microsystems was used for imaging immunofluorescent sections. IgG was used as the negative control.

For immunohistochemistry (IHC), the general procedure was similar to immunofluorescence, the endogenous peroxidase was inactivated with $3 \%$ hydrogen peroxide before antigen retrieval. Anti-ISL1 antibody was applied overnight at $4{ }^{\circ} \mathrm{C}$. A biotinylated goat anti-mouse secondary antibody (1:200; 115-065-146; Jackson ImmunoResearch, West Grove, PA, USA) was applied for $2 \mathrm{~h}$ at room temperature followed by streptavidin peroxidase (1:200; 123-065-021; Jackson ImmunoResearch, West Grove, PA, USA) for $2 \mathrm{~h}$. Diaminobenzidine (D4293; Sigma, St. Louis, MO, USA) and $0.1 \% \mathrm{H}_{2} \mathrm{O}_{2}$ were used for detecting peroxidase activity.

\section{Detection of proliferation and apoptotic cells}

To detect the proliferation of urethral cells, pregnant mice were injected intraperitoneally with $\mathrm{BrdU}(100 \mathrm{mg} /$ $\mathrm{kg}$ body weight) $4 \mathrm{~h}$ before sacrifice. Embryos were removed and processed for immunofluorescence staining, following the procedure described above. Terminal deoxynucleotidyl transferase dUTP nick-end labeling (TUNEL) assay for the detection of apoptotic cells was performed with the In Situ Apoptosis Detection Kit (Roche Applied Science) according to the manufacturer's instructions. Four embryos of each group were included for the analysis, and six sections of each embryo were examined for the proliferation and apoptosis.

\section{Transient transfection and Dual-Luciferase reporter assays}

The Shh, Fgfr2, and Fgf8 promoter fragment (0 to $-2000 \mathrm{bp}$ ) was cloned from mouse genomic DNA and inserted into the pGL3.0 vector (E1751, Promega). Isl1 was amplified using the primers containing the NheIXhoI restriction sites and inserted into the pcDNA3.1 vector. An empty luciferase reporter vector was used as a control. The primer sequence is in Supplementary Table S3.

The human embryonic kidney 293FT cells were maintained in Dulbecco's modified Eagle's medium supplemented with $10 \%$ fetal bovine serum and $1 \%$ penicillin-streptomycin at $37^{\circ} \mathrm{C}$ with $5 \% \mathrm{CO}_{2}$. Cells were transfected with Isl1 expression vector, Fgf8, Fgfr2, Shh luciferase reporter vector, and pTK-Ranilla vector using Lipofectamine 2000 (Invitrogen) according to the manufacturer's instruction. Twenty four hours post transfection, luciferase activity was measured by using DualLuciferase Reporter Assay Kit (E1910; Promega) on a Modulus II Microplate Multimode Reader (Turner Biosystems, Sunnyvale, CA, USA). The values were normalized against Renilla luciferase activity. At least four independent experiments were performed.

\section{Yeast one-hybrid assay}

The Fgf8, Fgfr2, Shh promoter fragment (0 to -2000 bp) was inserted into the pLacZi vector and Isl1 fragments was inserted into the $\mathrm{pB} 42 \mathrm{AD}$ vector as described previously. The primer sequence is in Supplementary Table S4. 
Plasmids for $\mathrm{pB} 42 \mathrm{AD}$ DNA-binding domain fusions (BD) were co-transformed with the pLacZi:LacZ reporter gene plasmid (AD) into the yeast strain EGY48 using standard transformation techniques. Transformants were grown on proper dropout plates containing X-gal (5-bromo-4chloro-3-indolyl- $\beta$-D-galactopyranoside) for color development. Yeast transformation and liquid assay were conducted as described in the Yeast Protocols Handbook (Clontech). At least four independent experiments were performed.

\section{Statistics}

The results were expressed as means \pm SEM of at least four independent experiments. The differences among groups were determined using a Student's $t$ test or a oneway analysis of variance. $P<0.05$ was considered to be statistically significant.

\section{Results}

\section{Is/1 expression in the developing mouse urethra}

First, we examined Isl1 messenger RNA (mRNA) levels in developing male mouse urethra from E12.5 to adult by using qPCR. Results showed that Isl1 mRNA levels were highest at E12.5, the earliest stage examined, decreasing by $40 \%$ at E13.5 and E15.5, and by $62.5 \%$ at E18.5 relative to levels observed at E12.5, followed by no significant changes up until adult stages (Fig. 1a). In addition, we investigated ISL1 expression by IHC, and results showed that ISL1 was weakly stained in the outgrowth at E11.5 (Fig. 1d, d1). At E12.5 and E15.5, ISL1 was strongly stained in most urethral epithelium (Fig. 1e, f, e1, f1) and around mesenchymal cells, but by E18.5, only a few ISL1positive cells were observed within the urethral epithelium (Fig. 1g, g1). This Isl1 expression pattern in during urethral development suggests that Isl1 might be involved in regulating urethral development.

\section{Is/1 expression is effectively ablated in $|s| 1^{M C M / D e l}$ mice}

To identify whether Isll was involved in regulating urethral development, the tamoxifen-inducible Cre/loxP system was utilized to knockout Isl1 in mouse as in our previous report ${ }^{25}$. Briefly, Isl1-MERCreMER males ${ }^{45}$ were used to mate with $I s l 1^{\text {loxP/loxP }}$ female mice ${ }^{48}$ to produce embryos carrying Isl1 mER-Cre-mER; Isl1 loxP/+ (Fig. 2a), where the $I s l 1$ promoter drives the expression of Cre. We then injected tamoxifen into pregnant dams at E9.5 to generate Isl1-inducible knockout embryos, hereafter referred to as $I s l 1^{M C M / D e l}$. Isl1 ${ }^{F /+}$ sibling embryos served as controls. Genotype of control and mutant embryos were detected by PCR (Fig. 2c). Western blot results showed that ISL1 protein levels in knockout mice decreased more than $90 \%$ compared with control embryos
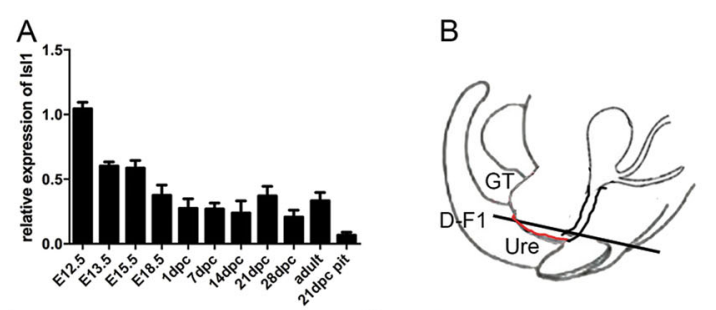

C
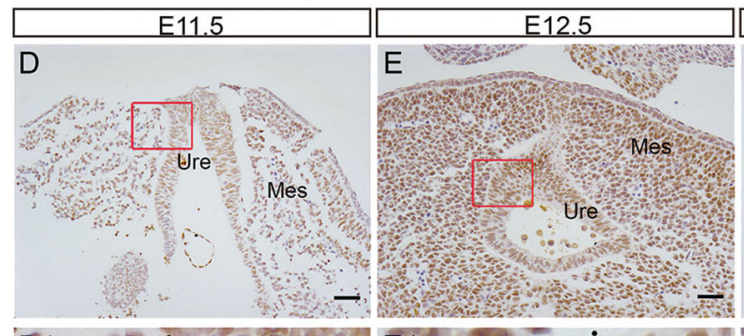

E15.5
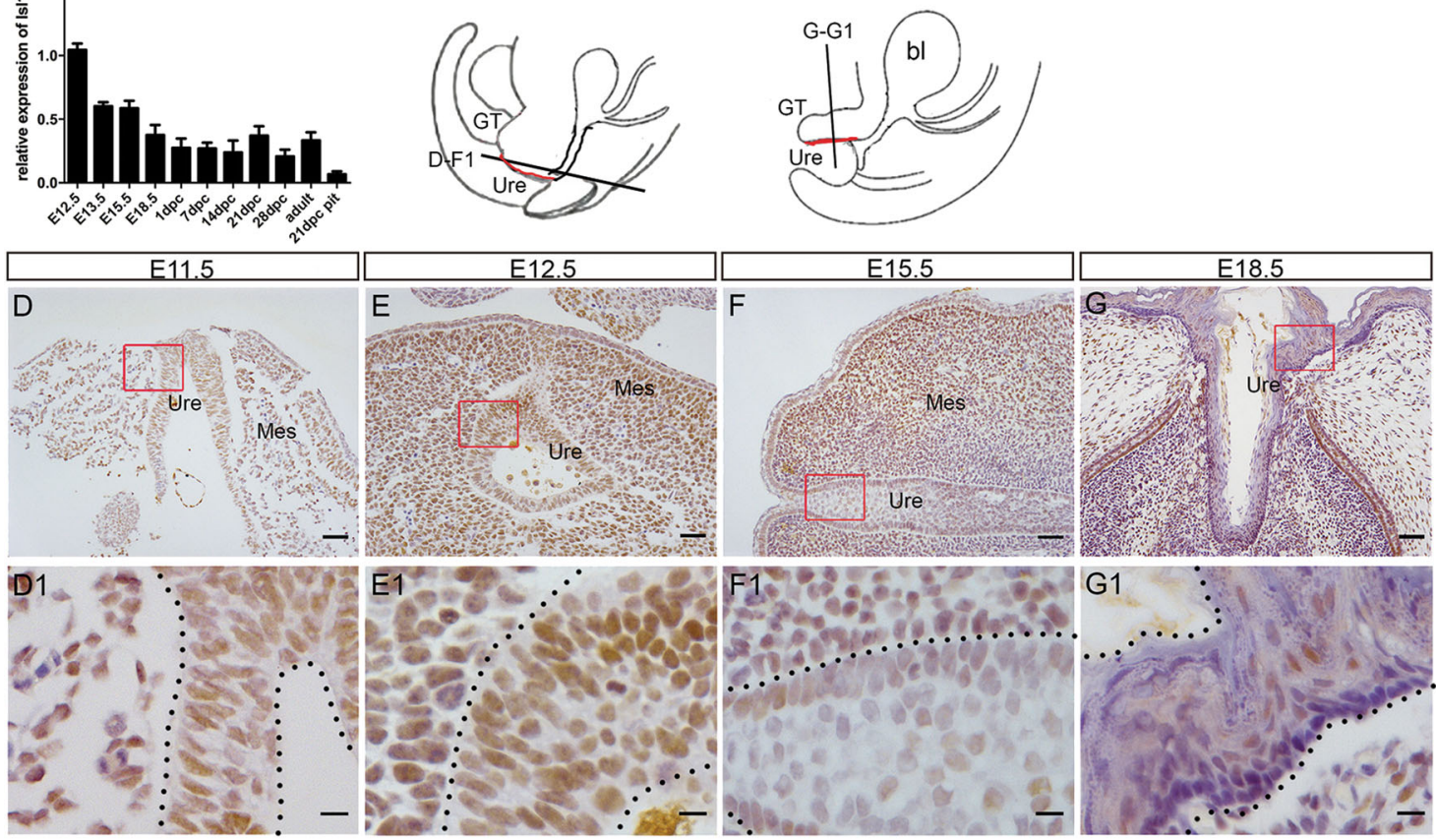

Fig. 1 Is/1 expression pattern during GT urethral development. a /s/1 mRNA relative expression levels at different developmental stages in male GTs. Twenty-one-day mice pituitaries served as a positive control. Each sample contained at least four independent replications. Data were presented as means \pm SEM. b, c Abridged general view of $\mathbf{d}-\mathbf{g} \mathbf{1}$ slice position. Red line represents the urethra. $\mathbf{d}-\mathbf{g} 1$ ISL1 localization in GTs at E11.5, E12.5, E15.5, and E18.5. Scale bars: $\mathbf{d}-\mathbf{g}, 50, \mu \mathrm{m} ; \mathbf{d 1} \mathbf{- g 1}, 10 \mu \mathrm{m}$. GT, genital tubercle; Mes, genital mesenchyme; Ure, urethra; bl, bladder 
A
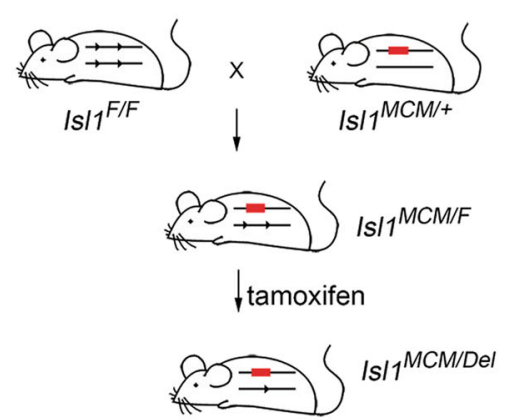

C

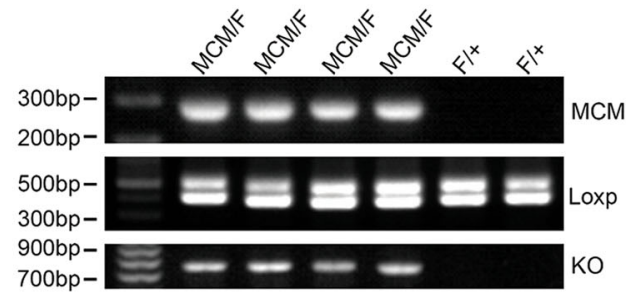

D
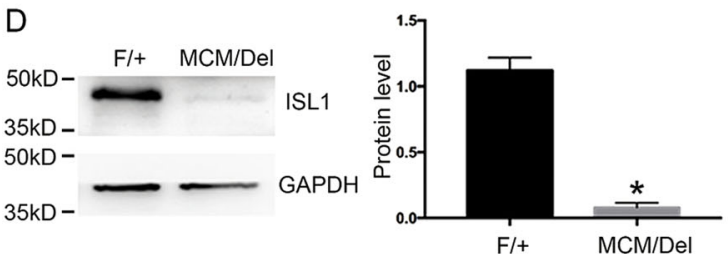
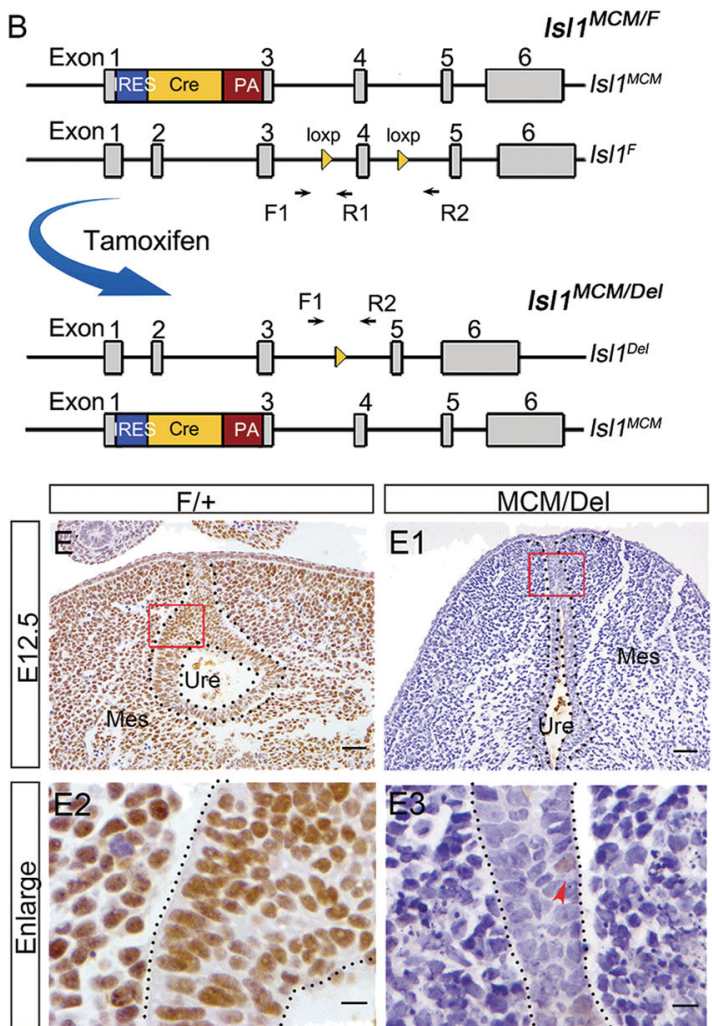

Fig. 2 Is/1 knocked down efficiency at E12.5 urethra in Is/1-inducible knockout embryos. a Schematic of inducible knockout mouse. b $/ s / 1$ gene knockout strategy and location of primers used for PCR verification of knockout genotype. $\mathbf{c}$ Genotype of $/ s / 1^{M C M / F}$ mutants and $/ s / 1^{\mathrm{F} /+}$ controls assayed by common PCR. $\mathbf{d}$ Representative image of Western blot detecting the knockdown efficiency of ISL1 protein. e-e3 ISL1 expression in urethral epithelium of $I s / 7^{\mathrm{MCM} / \mathrm{Del}}$ and control embryos. Scale bars: e-e1, $50 \mu \mathrm{m} ; \mathbf{e 2 - \mathbf { e }}, 10 \mu \mathrm{m}$. Red arrowheads representative ISL1-positive cells. Results were presented as means \pm SEM. Each test contained at least four independent replications for Western blot and $P C R$. ${ }^{*} P<0.05$

(Fig. 2d). IHC results demonstrated that ISL1 staining was reduced in urethral epithelium of $I s l 1^{M C M / D e l}$ mice compared to controls (Fig. 2e-e3). These data demonstrated that Isl1 was effectively down-regulated in urethral epithelium of $I s l 1^{M C M / D e l}$ embryos.

\section{Is/1 deletion disrupts urethral internalization}

To investigate effects of Isl1 on GT urethral development, morphological and histological examinations were performed at E15.5 and E18.5 for Isl1 ${ }^{\text {MCM/Del }}$ embryos and control littermates. Isl1 deletion resulted in hypoplasia of the GT in male and female embryos (Fig. 3), while GTs had developed with a urethral seam along the ventral midline in control male embryos at E15.5 (Fig. 3a). The overall size of mutant GTs was smaller than control GTs. In addition, the proximal urethral opening of mutants was both wider mediolaterally and extended farther proximodistally compared with controls (Fig. 3b). By E18.5, in Isl $1^{\text {MCM/Del }}$ male embryos, the fusion of the GT prepuce failed, which led to an ectopic opening (Fig. 3e, f).

Further histological analyses revealed that at E15.5, the proximal urethral plate of control embryos had separated medially to form a urethral tube (Fig. 3a2), whereas the distal end remained a bilaminar epithelial plate (Fig. 3a1). In $I s l 1^{M C M / D e l}$ mutants at the same stage, urethral tube epithelium was not detected proximally (Fig. 3b2), but the urethral remnant and a shallow groove on the ventral surface of the genital distal were observed (Fig. 3b1). By E18.5, Isl1 deletion resulted in the absence of internal urethra of male GTs (Fig. 3, compare e1 with f1, Fig. 3, compare e2 with f2).

Female Isl1 $1^{\text {MCM/Del }}$ mutants had hypoplasia similar to that observed in males, but effects on prepuce fusion were not as severe as those observed in male mutants either at E15.5 (Fig. 3c, d) or E18.5 (Fig. 3g, h). Isl1 deletion resulted in an open ventral urethra in females (Fig. 3, compare c2 with $\mathrm{d} 2$ at E15.5 and compare g2 with h2 at E18.5). Collectively, these data demonstrated that $I s l 1$ was crucial for urethral formation of GTs.

\section{Is/1 affects epithelial stratification and urethral cell differentiation}

Formation of the GT epithelium initiates as early as $\mathrm{E} 12.5^{49}$. In male embryos, histological examination 


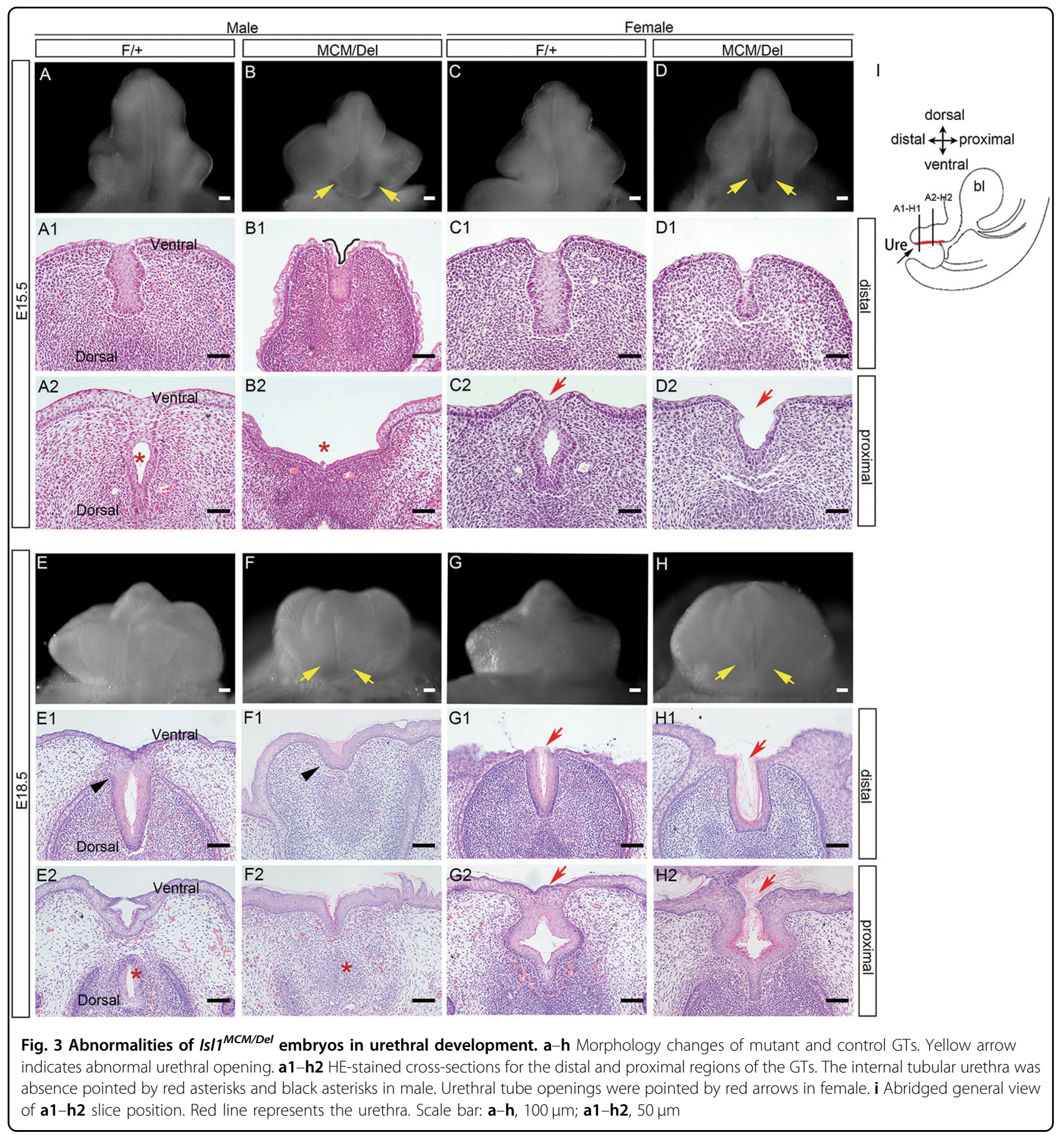

showed that, in control embryos, basal cells were columnar and elongated perpendicular to the basement membrane, intermediate cells were smaller, and apical cells were rounded or squamous. In contrast, urethral cells in male Isl1 ${ }^{\text {MCM/Del }}$ embryos were more homogenous, all cells being rounded, with no obvious orientation within the epithelium (Fig. 4a, b). Keratin 14 (K14), a differentiation marker, was not detected either in male controls or mutants (Fig. 4c, d). By E15.5, the GT epithelium in male controls was stratified in 4-6 cell layers, whereas in Isl1 ${ }^{M C M / D e l}$ embryos, GT epithelium was significantly thinner, being composed of 2-4 cell layers (Fig. 4e, f). In addition, in controls, K14 was evenly expressed in all urethral epithelial cells, but in $I s l 1^{\text {MCM/Del }}$ embryos, K14 was expressed only in urethral epithelium close to the lumen (Fig. 4g, h). Female embryos exhibited 


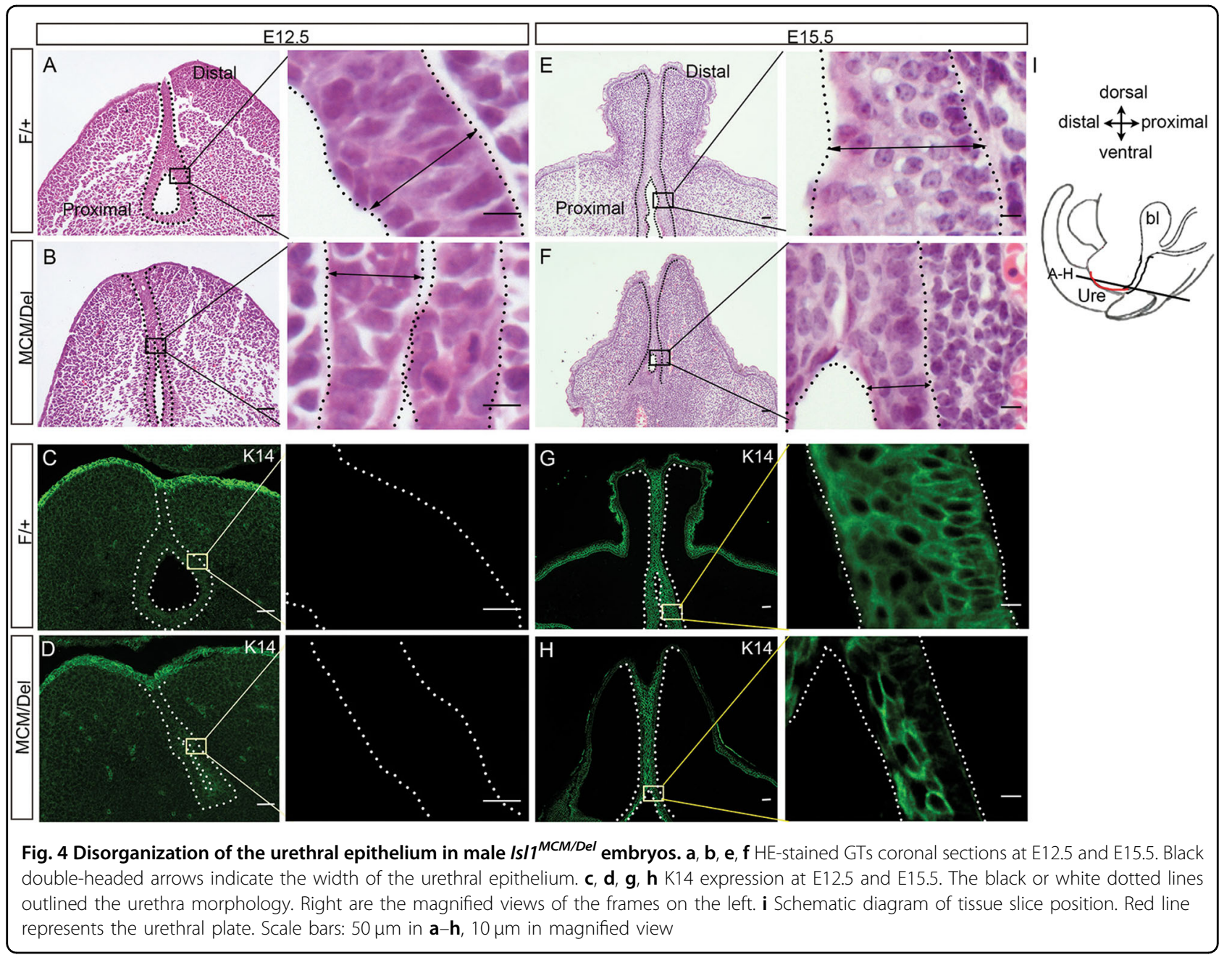

mutant phenotypes similar to those observed in males (data not shown). These results indicated that Isl1 affects epithelial stratification and urethral cell differentiation.

\section{Is/1 knockout enhances apoptosis of urethral epithelial cells}

To investigate the causes by which Isl 1 ablation resulted in reductions in urethral epithelial layers, we examined urethral epithelial cell apoptosis. Results showed that the proportion of TUNEL-positive cells within epithelial cells drastically increased in $I s l 1^{M C M / D e l}$ male embryos (28.3\%) compared with those in control embryos (6.3\%) at E12.5 (Fig. 5a, b, j). However, no TUNEL-positive cells were detected in urethral epithelium of controls or $I s l 1^{M C M / D e l}$ mutants at E15.5 (Fig. 5e, f, j). In addition, we analyzed mRNA levels of the anti-apoptosis gene $\mathrm{Bcl}-2$ and the proapoptosis gene $B a x$ by qPCR analysis of RNA isolated from whole GT tissue. Results showed that $\mathrm{Bax} / \mathrm{Bcl}-2$ ratios rose significantly both in E12.5 and E15.5 Isl ${ }^{\text {MCM/Del }}$ male embryos GTs relative to controls (Fig. 5l, m). Increased apoptosis indicated by the qPCR results at E15.5 were likely owing to inclusion of urethral mesenchymal cells, as we observed increased apoptosis in mesenchymal cells in mutants relative to controls at this stage (Fig. 5e, f).

To monitor cell proliferation, we performed BrdU labeling and immunostaining studies. In E12.5 male embryos, the proportion of BrdU-positive cells within total urethral epithelium was not significantly different between controls and mutants (49.1\% versus $50.3 \%$, Fig. 5c, d, k). The overall proportion of BrdU-positive cells significantly decreased in both controls and mutants in going from E12.5 to E15.5 (11.3\% and 15.7\%, respectively, Fig. $5 \mathrm{~g}, \mathrm{~h}, \mathrm{k}$ ), consistent with a decreased proliferation rate at the later stage ${ }^{10}$.

Furthermore, we analyzed effects of Isl1 expression on cell cycle genes, including Cyclin A1, Cyclin D2, Cyclin E1, $D p 1$, and E2f1 in GTs of the Isl1 ${ }^{M C M / D e l}$ and control male embryos at E12.5 and E15.5. Results showed mRNA levels of these genes exhibited no significant differences between $I s l 1^{\text {MCM/Del }}$ and control male embryos (Fig. 5n, o). Similar results were observed for female embryos (data not shown). 


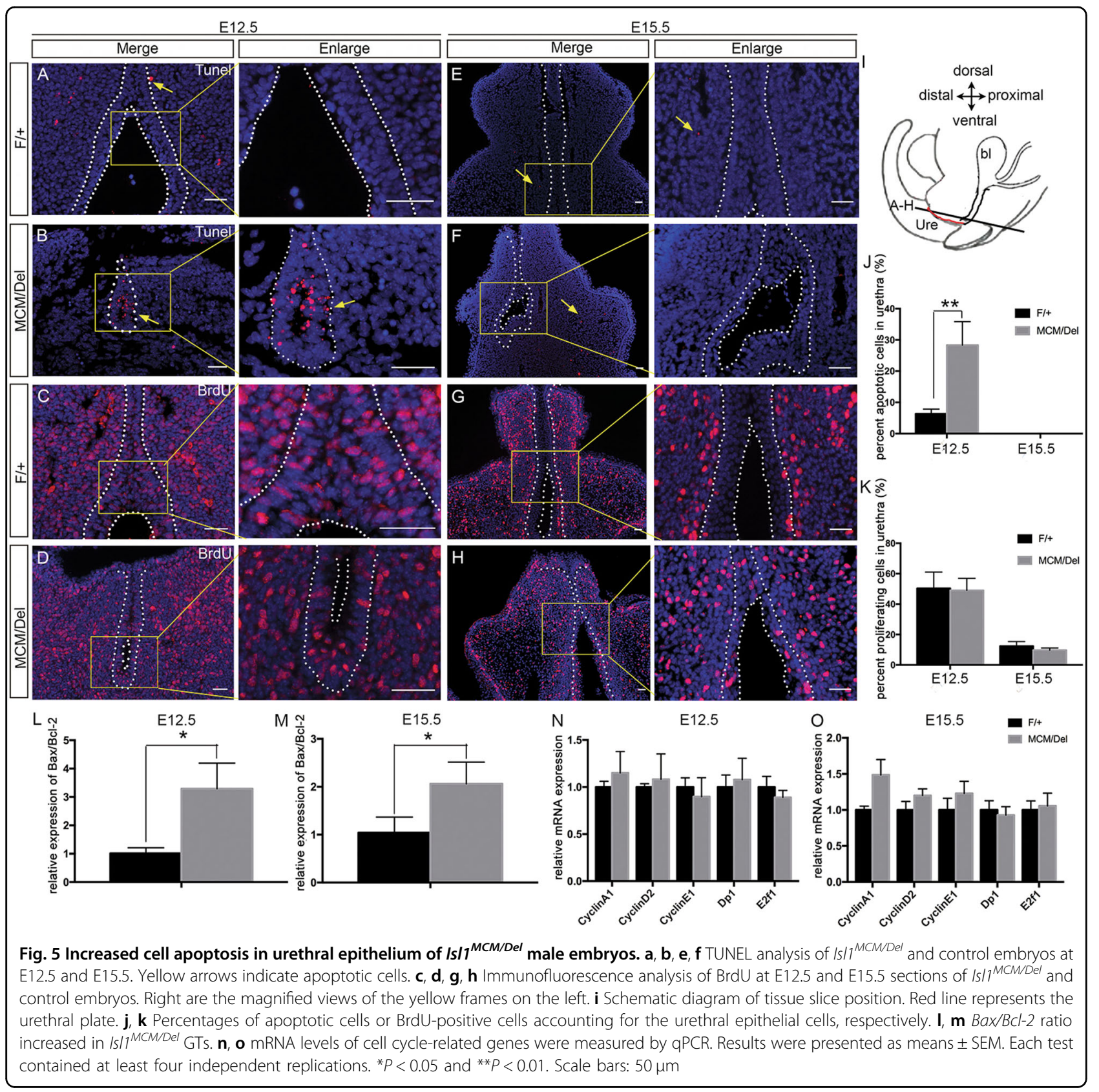

These results indicated that Isl1 ablation enhanced apoptosis of urethral epithelial cells at E12.5, but did not have significant effects on cell proliferation.

\section{ISL1 maintains urethral epithelium by targeting Shh}

To find potential direct downstream targets of ISL1 affecting differentiation and apoptosis of urethral epithelial cells in mutants, we examined expression of genes required for development of urethral epithelium, including Lef1, Bmp7, Msx1, Fgf8, Shh, Fgfr2, and Noggin, by qPCR analyses of mRNA from mutants and controls. Results showed that levels of Shh mRNA were decreased in GTs of $I s l 1^{M C M / D e l}$ embryos relative to controls at E12.5 and E15.5, whereas Fgfr2 and Fgf8 mRNA levels were significantly increased. Isl1 ablation did not have significant effects on mRNA levels of Lef1, Bmp 7, Msx 1 either at E12.5 or E15.5. At E15.5, Noggin mRNA levels were significantly lower in $I s l 1^{M C M / D e l}$ embryos when compared with controls (Fig. 6a, b). These data suggested that $S h h, F g f 8$, and $F g f r 2$ were potential direct downstream targets of ISL1.

To investigate whether Shh, Fgf8, and/or Fgfr2 were direct target genes of ISL1 regulating urethral epithelium development, we performed Dual-Luciferase reporter and 


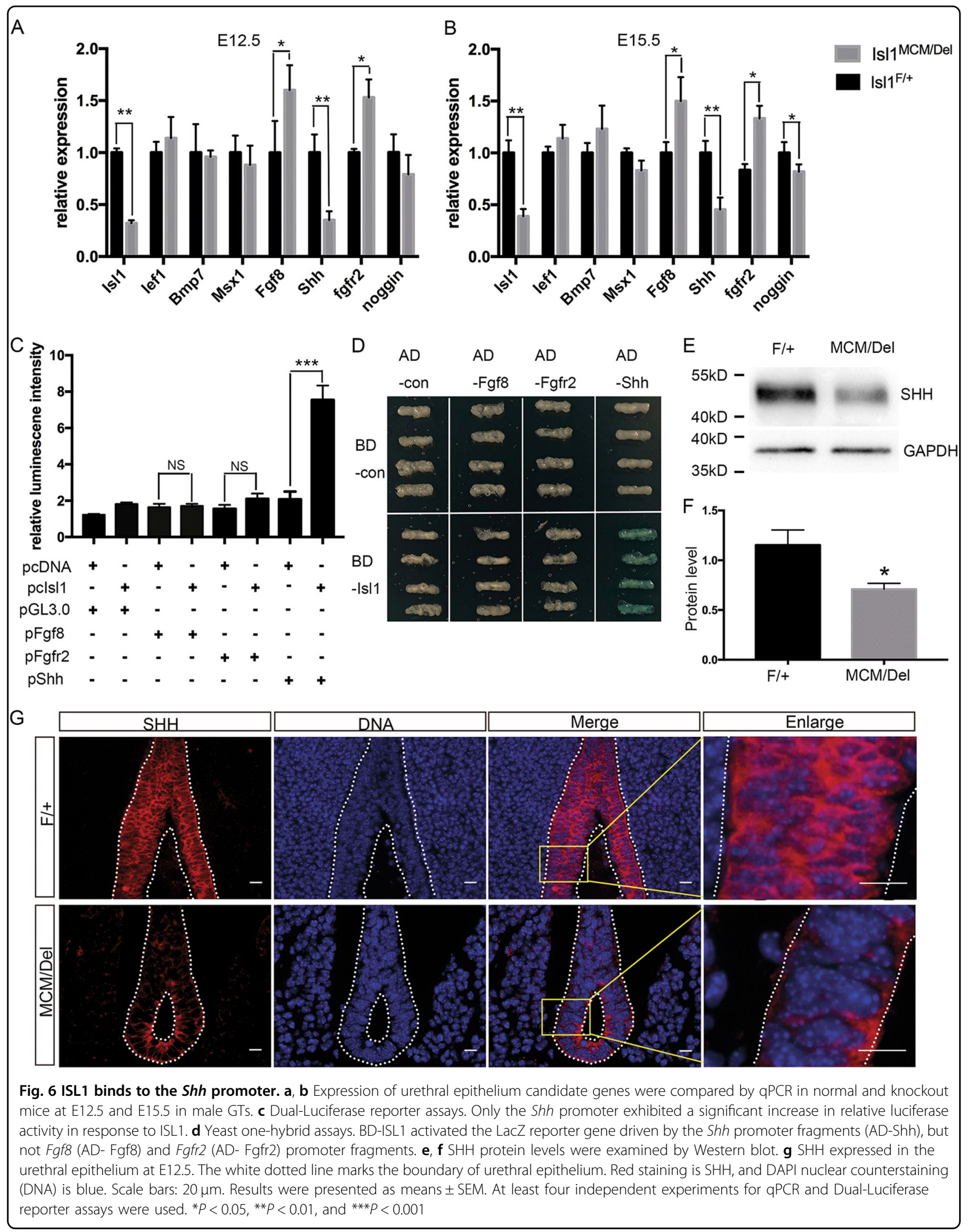


yeast one-hybrid assays. Promoter regions of Shh, Fgf8, and Fgfr2 were cloned, selecting $1-2000 \mathrm{bp}$ upstream of the $5^{\prime}$-untranslated regions as core promoter regions. Bioinformatics analyses revealed potential target sites for ISL1 (ATTA/TAAT) in promoters of all three genes. Results of Dual-Luciferase reporter assays demonstrated that ISL1 overexpression enhanced activity of the Shh promoter-Luciferase reporter $\sim 3$-fold, while $F g f 8$ and Fgfr2 promoter-Luciferase reporter activities exhibited no significant differences (Fig. 6c). Similarly, yeast one-hybrid assays showed that the ISL1 only activated the Shh promoter (Fig. 6d). In addition, Western blot results showed that SHH protein levels decreased by $40 \%$ in $I s l 1^{M C M / D e l}$ embryos (Fig. 6e, f) relative to controls. Additionally, immunofluorescence analyses showed that $\mathrm{SHH}$ was expressed in urethral epithelial cells at E12.5, and ISL1 and $\mathrm{SHH}$ proteins were co-expressed within cells of the urethral epithelium. SHH protein decreased after Isl1 depletion, which was consistent with qPCR and Western blot data (Fig. 6g). Together, these data supported Shh as a direct target of ISL1, indicating that Isl1 affects urethral epithelium development via $S h h$.

\section{Discussion}

Expression and function of the LIM homeobox protein ISL1 has been extensively studied in the nervous system $^{23,26,50}$, digestive system ${ }^{24,25}$, and endocrine glands ${ }^{39,40}$. Results of the present study demonstrated that Isl1 was expressed in urethral epithelial cells throughout urethral development, and that ablation of Isl1 just prior to GT development in $I s l 1^{M C M / D e l}$ mutants led to GT morphological abnormalities and urethral hypoplasia.

Results indicated that Isl1 was highly expressed in urethral epithelial cells and affected urethral formation throughout GT ontogeny. However, there is a report that conditional knockout mutants of Isl1 using Tbx4-Cre showed GT morphological abnormalities, but no significant effect on urethral development ${ }^{41}$. Tbx4-Cre is expressed in mesenchymal cells beginning in early embryogenesis ${ }^{51}$; thus, Isl1 was specifically knocked out in mesenchymal cells. In the study presented here, we used Isl1-MERCreMER ${ }^{45}$ induced with tamoxifen at E9.5 as the development of the urinary tract occurs after E10.5 and Isl1-null embryos die between E7.5 and E9.5 $5^{45}$. In our model, Isl1 was knocked out both in mesenchymal and urethral epithelial cells from E9.5. Our results showed that Isl1 deficiency resulted in GT morphological abnormalities and complete absence of the male urethra at E18.5. In support, our results are in agreement with reports that Isl1 is expressed in mesenchymal cells derived from mesoderm, which subsequently forms the prepuce $^{41,42}$.Thus, ISL1 is crucial for several aspects of urethral formation, as in the nervous system ${ }^{23,24,50}$, digestive system $^{25,26}$, and endocrine glands systems ${ }^{39,40}$.
In addition, results of this study revealed that ISL1 affects urethra formation by influencing cell survival and differentiation of the epithelial cells. Histological examination revealed reduced layers of stratified urethral epithelial cells in mutants at E12.5 and E15.5, with cells closer to the lumen being more elongated in Isl1-knockout mice. In addition, the proportion of TUNEL-positive cells within epithelial cells was over four times higher in $I s l 1^{M C M / D e l}$ male embryos than in the control in the early stage of urethra formation. However, Ching et al. ${ }^{41}$ reported that conditional knockout of Isl 1 in GT mesenchymal cells resulted in reduced apoptosis and had no significant effects on cell proliferation in $\mathrm{GT}^{51}$.

Shh plays important roles in regulating formation and development of multiple organs ${ }^{52-57}$, including urethral development ${ }^{2,58}$. Shh is specifically expressed in urethral epithelial cells, and Shh knockout leads to abnormal cell cycle $^{59}$ of the urinary tract, increased cell death, and results in urethral hypoplasia, ${ }^{2,58}$, similar to phenotypes observed with the Isl1 $1^{M C M / D e l}$ mice model presented here. These observations suggest that Isl1 may affect GT development by regulating expression of $S h h$. In support, our results showed that both Isl1 and Shh were expressed in all urethral epithelial cells. In addition, results of DualLuciferase reporter and yeast one-hybrid assays demonstrated that the Shh promoter contained consensus binding sites for ISL1, and that ISL1 overexpression enhanced activity of the $S h h$ promoter. These results are consistent with ISL1 influencing urethral epithelium development by regulation of Shh expression.

Urethral hypoplasia is a common birth defect in humans, yet its etiology and pattern of onset are largely unknown. There are many factors that cause urethral hypoplasia, including environment, diet, and heredity factors. Several studies have detected significant changes in Isl1 in human urethral hypoplasia samples compared to the normal sample by sequencing ${ }^{42,60}$. Draaken et al. ${ }^{42}$ reported that Isl1 DNA was significantly decreased in BEEC patients, suggesting that Isl1 plays a role in BEEC. Our laboratory studies showed that Isl1 affected stomach pyloric sphincter development. We investigated the role of Isl1 in the urethral sphincter, which may lead to BEEC. In addition, we tested the urethral sphincter and bladder of $I s l 1^{M C M / D e l}$ mutants, and no significant abnormalities were found until E18.5 (data not shown). It may be that Isl $1^{\text {MCM/Del }}$ mutants are not suitable for BEEC studies, but Isl ${ }^{M C M / D e l}$ mutants can be used as an animal model to study diseases related to urethral hypoplasia.

Lastly, we presented the novel mechanism of Isl1 regulating urethral development (Fig. 7). In brief, Isl1 affects urethral epithelium fate by directly targeting $S h h$ promoter, subsequently affects commitment of progenitor cell, and enhance cell death. These findings are important for our 


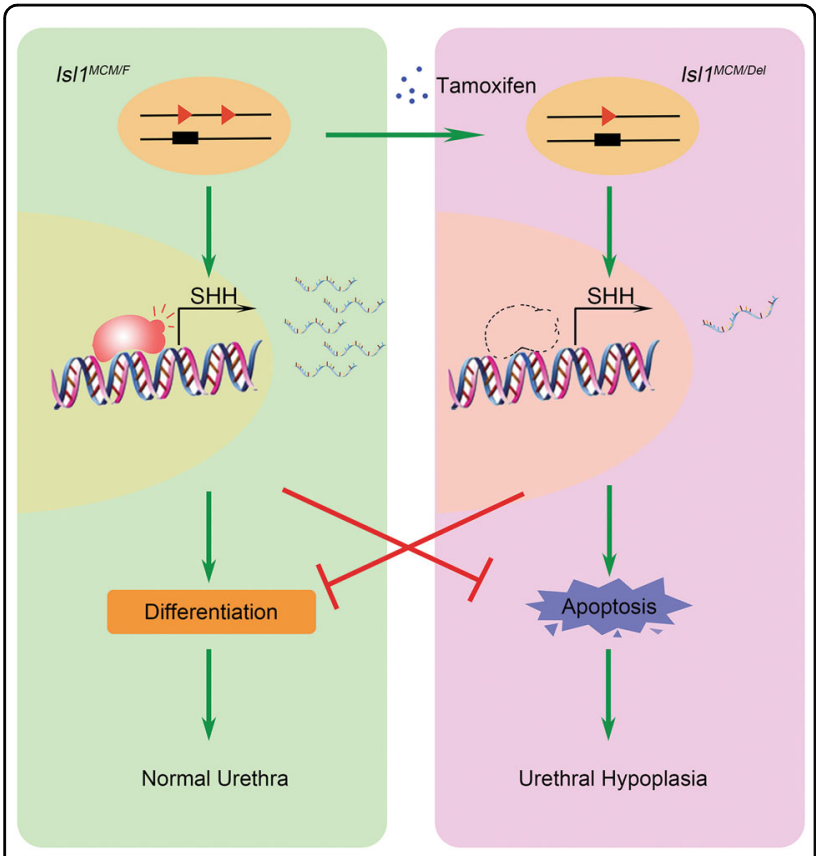

Fig. 7 Model of $I s / 1$ function in mice developing urethral

epithelium. Tamoxifen-induced Is/1 DNA recombine and decreased ISL1 protein level. Insufficient ISL1 protein bind to the Shh gene promoter lead to failure of Shh transcription activation. This affects commitment of urethral epithelial cell and increasing apoptosis then resulting in hypoplasia. The green line indicates positive regulation and the red line indicates negative regulation

understanding of diseases resulting from abnormalities of urethral development.

\section{Acknowledgements}

This work is supported by the National Key Research and Developmental Program of China (2018YFC1003504), the Natural Science Foundation of China $(31430083,31772692)$ and the Project of the Priority Academic Program Development of Jiangsu Higher Education Institutions (PAPD).

\section{Author details}

'State Key Laboratory of Agrobiotechnology, College of Biological Sciences, China Agricultural University, 100193 Beijing, People's Republic of China. ${ }^{2}$ College of Veterinary Medicine, Yangzhou University, 225009 Yangzhou, Jiangsu, People's Republic of China. ${ }^{3}$ Skaggs School of Pharmacy, University of California San Diego, 9500 Gilman Drive, La Jolla, CA 92093, USA. ${ }^{4}$ Key Laboratory of Human Disease Comparative Medicinelnstitute of Laboratory Animal Science, Chinese Academy of Medical Science and Comparative Medical Center, Peking Union Medical College, 100021 Beijing, People's Republic of China

\section{Conflict of interest}

The authors declare that they have no conflict of interest.

\section{Publisher's note}

Springer Nature remains neutral with regard to jurisdictional claims in published maps and institutional affiliations.

Supplementary Information accompanies this paper at (https://doi.org/ 10.1038/s41419-019-1952-z).
Received: 10 June 2019 Revised: 25 August 2019 Accepted: 3 September 2019

Published online: 26 September 2019

\section{References}

1. Haraguchi, R. et al. Molecular analysis of external genitalia formation: the role of fibroblast growth factor (Fgf) genes during genital tubercle formation. Development 127, 2471-2479 (2000).

2. Lin, C. et al. Temporal and spatial dissection of Shh signaling in genital tubercle development. Development 136, 3959-3967 (2009).

3. Qian, C. et al. Conditional deletion of platelet derived growth factor receptor alpha (Pdgfra) in urorectal mesenchyme causes mesenchyme apoptosis and urorectal developmental anomalies in mice. Cell Death Differ 26, 1396-1410 (2019).

4. Matsushita, S. et al. Regulation of masculinization: androgen signalling for external genitalia development. Nat. Rev. Urol. 15, 358-368 (2018).

5. Baskin, L. et al. Urethral seam formation and hypospadias. Cell Tissue Res. 305, 379-387 (2001).

6. Suzuki, K. et al. Regulation of outgrowth and apoptosis for the terminal appendage: external genitalia development by concerted actions of BMP signaling [corrected]. Development 130, 6209-6220 (2003).

7. Perriton, C. L., Powles, N., Chiang, C., Maconochie, M. K. \& Cohn, M. J. Sonic hedgehog signaling from the urethral epithelium controls external genital development. Dev. Biol. 247, 26-46 (2002).

8. Cohn, M. J. Development of the external genitalia: conserved and divergent mechanisms of appendage patterning. Dev. Dyn. 240, 1108-1115 (2011).

9. Herrera, A. M. \& Cohn, M. J. Embryonic origin and compartmental organization of the external genitalia. Sci. Rep. 4, 6896 (2014).

10. Petiot, A., Perriton, C. L., Dickson, C. \& Cohn, M. J. Development of the mammalian urethra is controlled by Fgfr2-IIIb. Development 132, 2441-2450 (2005).

11. Cunha, G. R., Sinclair, A., Risbridger, G., Hutson, J. \& Baskin, L. S. Current understanding of hypospadias: relevance of animal models. Nat. Rev. Urol. 12 271-280 (2015)

12. Shiroyanagi, Y. et al. Urothelial sonic hedgehog signaling plays an important role in bladder smooth muscle formation. Differentiation 75, 968-977 (2007).

13. Cheng, W. et al. Sonic hedgehog mediator Gli2 regulates bladder mesenchymal patterning. J. Urol. 180, 1543-1550 (2008).

14. Haraguchi, R. et al. The hedgehog signal induced modulation of bone morphogenetic protein signaling: an essential signaling relay for urinary tract morphogenesis. PLoS ONE 7, e42245 (2012).

15. Ng, R. C. et al. Dysregulation of Wht inhibitory factor 1 (Wif1) expression resulted in aberrant Wnt-b-catenin signaling and cell death of the cloaca endoderm, and anorectal malformations. Cell Death Differ. 21, 978-989 (2014).

16. Morgan, E. A. Loss of Bmp7 and Fgf8 signaling in Hoxa13-mutant mice causes hypospadia. Development 130, 3095-3109 (2003).

17. Seifert, A. W., Yamaguchi, T. \& Cohn, M. J. Functional and phylogenetic analysis shows that Fgf8 is a marker of genital induction in mammals but is not required for external genital development. Development 136, 2643-2651 (2009).

18. Gredler, M. L., Seifert, A. W. \& Cohn, M. J. Tissue-specific roles of Fgfr2 in development of the external genitalia. Development 142, 2203-2212 (2015).

19. Ikeda, Y. et al. Fgfr2 is integral for bladder mesenchyme patterning and function. Am. J. Physiol. Ren. Physiol. 312, F607-F618 (2017).

20. Lin, C., Yin, Y., Long, F. \& Ma, L. Tissue-specific requirements of beta-catenin in external genitalia development. Development 135, 2815-2825 (2008).

21. Lin, C. et al. Delineating a conserved genetic cassette promoting outgrowth of body appendages. PLoS Genet. 9, e1003231 (2013).

22. Miyagawa, S. et al. Disruption of the temporally regulated cloaca endodermal beta-catenin signaling causes anorectal malformations. Cell Death Differ. $\mathbf{2 1}$ 990-997 (2014).

23. Kim, K. T. et al. ISL1-based LIM complexes control Slit2 transcription in developing cranial motor neurons. Sci. Rep. 6, 36491 (2016).

24. Zhang, Q. et al. Temporal requirements for ISL1 in sympathetic neuron proliferation, differentiation, and diversification. Cell Death Dis. 9, 247 (2018).

25. Li, Y. et al. LIM homeodomain transcription factor Isl1 directs normal pyloric development by targeting Gata3. BMC Biol. 12, 25 (2014).

26. Guo, T. et al. ISL1 predicts poor outcomes for patients with gastric cancer and drives tumor progression through binding to the ZEB1 promoter together with SETD7. Cell Death Dis. 10, 33 (2019). 
27. Suzuki, K. et al. Reduced BMP signaling results in hindlimb fusion with lethal pelvic/urogenital organ aplasia: a new mouse model of sirenomelia. PLOS ONE 7, e43453 (2012).

28. Tahara, N. et al. Gata6 restricts $|s| 1$ to the posterior of nascent hindlimb buds through Isl1 cis-regulatory modules. Dev. Biol. 434, 74-83 (2018).

29. Yang, L. et al. Isl1Cre reveals a common Bmp pathway in heart and limb development. Development 133, 1575-1585 (2006).

30. Gao, R. et al. Pioneering function of $|s| 1$ in the epigenetic control of cardiomyocyte cell fate. Cell Res. 29, 486-501 (2019).

31. Witzel, H. R. et al. The LIM protein Ajuba restricts the second heart field progenitor pool by regulating Isl1 activity. Dev. Cell 23, 58-70 (2012).

32. Lin, L. et al. Beta-catenin directly regulates Islet1 expression in cardiovascular progenitors and is required for multiple aspects of cardiogenesis. Proc. Natl Acad. Sci. USA 104, 9313-9318 (2007).

33. Laugwitz, K. L., Moretti, A., Caron, L., Nakano, A. \& Chien, K. R. Islet1 cardiovascular progenitors: a single source for heart lineages? Development 135 , 193-205 (2008).

34. Elshatory, Y., Deng, M., Xie, X. \& Gan, L. Expression of the LIM-homeodomain protein $|s| 1$ in the developing and mature mouse retina. J. Comp. Neurol. $\mathbf{5 0 3}$ 182-197 (2007)

35. Bejarano-Escobar, R. et al. Expression and function of the LIM-homeodomain transcription factor islet- 1 in the developing and mature vertebrate retina. Exp. Eye Res. 138, 22-31 (2015).

36. Ediger, B. N. et al. Islet-1 Is essential for pancreatic beta-cell function. Diabetes 63, 4206-4217 (2014).

37. Yan, C. et al. Protein Inhibitor of activated STAT Y (PIASy) regulates insulin secretion by interacting with LIM homeodomain transcription factor Isl1. Sci. Rep. 6, 39308 (2016).

38. Wu, Y. et al. LIM homeodomain transcription factor Isl-1 enhances follicle stimulating hormone-beta and luteinizing hormone-beta gene expression and mediates the activation of leptin on gonadotropin synthesis. Endocrinology 151, 4787-4800 (2010).

39. Zhang, J. et al. LIM homeobox transcription factor $|s| 1$ is required for melatonin synthesis in the pig pineal gland. J. Pineal Res. 65, e12481 (2018).

40. Qiu, J. et al. MicroRNA-7 inhibits melatonin synthesis by acting as a linking molecule between leptin and norepinephrine signaling pathways in pig pineal gland. J. Pineal Res. 66, e12552 (2019).

41. Ching, S. T. et al. Isl1 mediates mesenchymal expansion in the developing external genitalia via regulation of Bmp4, Fgf10 and Wnt5a. Hum. Mol. Genet 27, 107-119 (2018).

42. Draaken, M. et al. Genome-wide association study and meta-analysis identify ISL1 as genome-wide significant susceptibility gene for bladder exstrophy. PLoS Genet. 11, e1005024 (2015).

43. Zhang, R. et al. ISL1 is a major susceptibility gene for classic bladder exstrophy and a regulator of urinary tract development. Sci. Rep. 7, 42170 (2017).
44. Kaku, Y. et al. Islet1 deletion causes kidney agenesis and hydroureter resembling CAKUT. J. Am. Soc. Nephrol. 24, 1242-1249 (2013).

45. Laugwitz, K. L. et al. Postnatal isl1+ cardioblasts enter fully differentiated cardiomyocyte lineages. Nature 433, 647-isl653 (2005).

46. Tunster, S. J. Genetic sex determination of mice by simplex PCR. Biol. Sex Differ. 8, 31 (2017).

47. Alasaad, S. et al. HotSHOT Plus ThermalSHOCK, a new and efficient technique for preparation of PCR-quality mite genomic DNA. Parasitol. Res. 103 1455-1457 (2008)

48. Sun, Y. et al. A central role for Islet1 in sensory neuron development linking sensory and spinal gene regulatory programs. Nat. Neurosci. 11, 1283-1293 (2008).

49. Georgas, K. M. et al. An illustrated anatomical ontology of the developing mouse lower urogenital tract. Development 142, 1893-1908 (2015).

50. Huber, $\mathrm{K}$. et al. The LIM-Homeodomain transcription factor Islet-1 is required for the development of sympathetic neurons and adrenal chromaffin cells. Dev. Biol. 380, 286-298 (2013).

51. Luria, V., Krawchuk, D., Jessell, T. M., Laufer, E. \& Kania, A. Specification of motor axon trajectory by ephrin-B:EphB signaling: symmetrical control of axonal patterning in the developing limb. Neuron 60, 1039-1053 (2008).

52. Zhang, L. et al. Sonic hedgehog signaling pathway mediates cerebrolysin-improved neurological function after stroke. Stroke 44, 1965-1972 (2013).

53. Qin, S. et al. Downregulation of sonic hedgehog signaling in the hippocampus leads to neuronal apoptosis in high-fat diet-fed mice. Behav. Brain Res. 367, 91-100 (2019).

54. Wang, Y., Peng, Q., Jia, H. \& Du, X. Prognostic value of hedgehog signaling pathway in digestive system cancers: a systematic review and meta-analysis. Cancer Biomark. 16, 71-79 (2016).

55. Konstantinou, D., Bertaux-Skeirik, N. \& Zavros, Y. Hedgehog signaling in the stomach. Curr. Opin. Pharmacol. 31, 76-82 (2016).

56. Jimenez-Caliani, A. J. et al. aE-catenin is a positive regulator of pancreatic islet cell lineage. Differ. Cell Rep. 20, 1295-1306 (2017).

57. Mfopou, J. K., De Groote, V., Xu, X., Heimberg, H. \& Bouwens, L. Sonic hedgehog and other soluble factors from differentiating embryoid bodies inhibit pancreas development. Stem Cells 25, 1156-1165 (2007).

58. Miyagawa, S. et al. Dosage-dependent hedgehog signals integrated with Wnt/beta-catenin signaling regulate external genitalia formation as an appendicular program. Development 136, 3969-3978 (2009).

59. Seifert, A. W., Zheng, Z., Ormerod, B. K. \& Cohn, M. J. Sonic hedgehog controls growth of external genitalia by regulating cell cycle kinetics. Nat. Commun. 1, 23 (2010).

60. Arkani, S. et al. Evaluation of the ISL1 gene in the pathogenesis of bladder exstrophy in a Swedish cohort. Hum. Genome Var. 5, 18009 (2018). 